Mots. Les langages du politique

$123 \mid 2020$

De la racine à l'extrémisme : discours des radicalités politiques et sociales

\title{
Les discours des radicalités politiques et sociales
}

Political and social radicality in speech

Los discursos de las radicalidades políticas y sociales

Valérie Bonnet, Béatrice Fracchiolla, Lilian Mathieu et Camille Noûs

\section{OpenEdition \\ Journals}

Édition électronique

URL : https://journals.openedition.org/mots/26557

DOI : $10.4000 /$ mots. 26557

ISBN : 979-10-362-0250-6

ISSN : 1960-6001

Éditeur

ENS Éditions

Édition imprimée

Date de publication : 10 septembre 2020

Pagination : 19-27

ISBN : $979-10-362-0249-0$

ISSN : 0243-6450

Référence électronique

Valérie Bonnet, Béatrice Fracchiolla, Lilian Mathieu et Camille Noûs, «Les discours des radicalités politiques et sociales », Mots. Les langages du politique [En ligne], 123 | 2020, mis en ligne le 01 janvier 2021, consulté le 04 janvier 2023. URL : http://journals.openedition.org/mots/26557 ; DOl : https:// doi.org/10.4000/mots. 26557 


\section{Les discours des radicalités politiques et sociales}

Le terme radicalité(s) a connu une recrudescence d'utilisations depuis quelques années. Ce numéro de la revue Mots entend traiter des radicalités, au pluriel, et non pas de la radicalité au sens où elle est souvent comprise en ce début de $x x I^{e}$ siècle, comme un phénomène qui serait essentiellement lié au djihadisme. Notre réflexion porte dans un premier temps sur ce qu'est la radicalité, dans une perspective générale, perspective nous permettant dans un second temps de définir les radicalités. Au travers des différents articles, nous avons ainsi cherché à déconstruire ce que l'on entend aujourd'hui par cette notion. En effet, si l'on se réfère au matériau linguistique, on constate que le vocable afférent, dans son acception politique, n'a pas encore trouvé sa place dans le dictionnaire ${ }^{1}$. Pour saisir les contours de ce terme passe-partout, on n'a d'autre choix que de passer par les lexèmes appartenant au même champ morphosémantique et répertoriés dans le corpus lexicographique. Ainsi, radical, e, est construit sur le bas latin radicalis, venant de radix, radicis qui signifie « racine». Tous les autres termes sont quant à eux construits plus ou moins directement à partir de ce terme, à la fois nom et adjectif : radical, radicalement, radicalisation, radicaliser, radicalisme, ou encore radical-socialisme, radical-socialiste. Les notions contenues dans l'ensemble des éléments de ce champ lexical en lien avec le sens politique renvoient à des éléments divers qui permettent d'en retracer l'évolution. Par exemple, l'appellation les radicaux renvoyait en 1820 aux républicains partisans de réformes dites «radicales», c'est-à-dire allant dans le sens de la démocratie politique et sociale. Au sens moderne, le parti radical, puis radical-socialiste, est constitué d'une fraction des républicains qui

1. Le Petit Robert de la langue française, 2018 (édition électronique) et Le Trésor de la langue française informatisé (TLFi), pour ne citer que ces deux ouvrages.

Université Toulouse 3, LERASS

valerie.bonnet@free.fr

Université de Lorraine, CREM

beatrice.fracchiolla@univ-lorraine.fr

CNRS-ENS de Lyon, Centre Max Weber

lilian.mathieu@ens-lyon.fr

Laboratoire Cogitamus / Cogitamus Laboratory

camille.nous@noussommesluniversite.fr 
se sont lancés à la conquête de la République après la chute du Second Empire; ils sont situés de nos jours au centre du champ politique et attestent un paradoxal retournement sémantique : censés incarner une forme d'intransigeance, ils constituent aujourd'hui une force politique marginale, contrainte pour survivre à des compromis avec des partis plus importants. En revanche, une doctrine est qualifiée de «radicale» lorsqu'elle va jusqu'au bout de ses conséquences, sans concession (extrémiste, intransigeante, intégriste, rigoriste 2 ). C'est sur cette base de signifié, à partir de l'adjectif radical, $e$, et non du substantif, que se comprend dans le contexte actuel la notion de "radicalité(s)» politique(s). Sans doute, d'ailleurs, éprouve-t-on le besoin de ce néologisme précisément parce que le nom correspondant s'est spécialisé dans un sens qui est pratiquement à l'opposé ("centriste») de ce que signifie l'adjectif radical et que reprend sémantiquement, donc, le substantif féminin radicalité.

En quelque sorte, cette double appartenance signifie à la fois une forme de retour aux sources (la notion de racine), qui est illustré dans ce numéro à travers l'article d'Ėve Gianoncelli consacré aux convergences entre les pensées de Jean-Claude Michéa et d'auteurs de la droite la plus conservatrice. Mais elle signifie aussi l'inverse de ce premier sens, à savoir une transformation extrême. Il en résulte une série de conséquences sur la manière d'envisager les sens en discours de radicalité(s) associé ici à politique(s). Le discours de la radicalité politique fonctionne ainsi également en polysémie et épaisseur de sens, comme le rappelle et l'illustre à ce propos le texte de Matthijs Gardenier et Angeliki Monnier, qui étudie le discours de justification de mobilisations vigilantistes hostiles aux migrants et leur rapport à la notion de radicalité. Dans cette mesure, la radicalité n'est pas forcément là où l'on pense qu'elle réside; c'est la poursuite d'une fin, déterminée, qui connaît différentes formes de «performances» (en termes de violence/non-violence, spectacle/ non-spectacle, discours/action).

En d'autres termes, et partant de la définition du discours de radicalité politique comme étant un discours de (dé)légitimation en même temps qu'un discours de confrontation, l'objet global de cette livraison de la revue Mots est de définir ce qu'est un discours radical et comment se traduit le discours des radicalités politiques, sans que cela ne relève nécessairement d'un discours de haine. Dans deux perspectives et contextes différents, les articles de Maria Gabriela Dascalakis-Labreze - portant sur les insultes animalières stigmatisant les principales personnalités politiques argentines, Mauricio Macri et Cristina Kirchner - et de M. Gardenier et A. Monnier - soulignant l'atténuation de l'agressivité des discours numériques affichés par les collectifs antimigrants - permettent de voir en quoi cet objectif est atteint.

2. En Allemagne, c'est le mot radikal qui est utilisé pour dire l'extrémisme politique : Die Rechtsradikalen, « extrême droite »; Die Linksradikalen, "extrême gauche ». 
Les différentes contributions retenues s'organisent en effet selon les quatre thématiques suivantes:

\section{Catégorisation}

Parler de «radicalités politiques», par l'expression même, sous-entend déjà que plusieurs personnes sont d'accord pour définir certains actes, événements, idées politiques comme «radicaux». Cela participe donc à la fois de l'autodéfinition ou de l'hétérodéfinition (les féministes sont jugées radicales là où elles peuvent considérer leurs positions comme relevant de la norme, et il en est de même de La Manif pour tous; voir Fracchiolla, 2015). En d'autres termes, la radicalité peut être considérée comme phénomène de réception ou comme phénomène d'émission. Ici intervient la double logique de nomination/catégorisation qui suppose cette radicalité comme assumée, revendiquée ou au contraire rejetée, stigmatisée. On perçoit donc comment un discours, une argumentation de confrontation s'organisent en miroir autour de l'idée même de radicalité. De fait, la radicalité peut autant être revendiquée comme un label valorisant - par ses connotations d'intransigeance, voire de rudesse politique, comme dans le cas du groupuscule néofasciste Unité radicale (Mathieu, 2003) - ou jouer comme un stigmate - comme lorsque le qualificatif de radical sert à disqualifier un féminisme considéré comme outrageusement hostile aux hommes ou trop exigeant. Les instrumentalisations croisées d'un tel label et ses éventuels retournements sémantiques - dont atteste par exemple l'existence, aux États-Unis, du courant autodéfini du féminisme radical - sont au cœur des luttes de définition et de catégorisation qui traversent les univers militants. Parce qu'elles modifient les perceptions de soi et des autres et, ce faisant, la place occupée dans un espace politique, ces luttes ne sont pas sans influence sur le processus de radicalisation lui-même, ainsi que le soulignent Annie Collovald et Brigitte Gaïti :

[...] les usages du label «radical» entrent dans l'analyse de la dynamique radicale elle-même : en modifiant les identités antérieurement attribuées aux mouvements désormais considérés et perçus comme radicaux, ils modifient également les lignes de conduite à tenir à leur égard et reconfigurent alors les solidarités et les alliances possibles, les stratégies pensables et, au bout du compte, leur position dans le jeu politique. (Collovald, Gaïti éd., 2006, p. 23)

Par ailleurs, l'appel à contributions à l'origine de ce dossier entendait questionner l'équivalence souvent établie entre un discours politiquement radical et un discours extrémiste. La radicalité semble en effet exclure la discussion ou la négociation, ce qui nous mène directement à une autre question, fondamentale, portant sur les modes argumentatifs de la radicalité et leurs formulations. Ceux-ci semblent très fortement se faire selon un registre 
chargé d'affects (formulation de la souffrance, de l'indignation, de la colère) ou encore par l'adoption d'une certaine posture de rupture, que la rhétorique définit comme un discours ne "se coulant [pas] dans l'autorité du consensus majoritaire» (Plantin, 2016, p. 194). Parmi les questions posées, soulignons par exemple celles de la radicalité construite sur le logos : la froide détermination, les logiques d'inversion de point de vue telles que mises en place par Malcolm X (voir Bonnet, 2017), le renvoi au discours expert des militants pour l'environnement (Bonnet, Geslin éd., 2019) ou encore, ici même, les discours de raison mis en œuvre par les collectifs étudiés par M. Gardenier et A. Monnier.

Il s'agissait également de savoir si la radicalité s'appuyait sur des modes et des genres privilégiés comme le pamphlet, la polémique (Amossy, 2014) et de questionner ses liens avec la violence verbale, la notion de montée en tension linguistique et discursive (Moïse et al. éd., 2008; Fracchiolla et al., 2013). Enfin, la question s'est également posée de savoir si la radicalité discursive se matérialisait obligatoirement par ce que Baruch Spinoza nommait les passions tristes (voir Quéré, 2017), si l'on songe à l'écriture inclusive, qui peut être prise comme telle dans certains discours, ou à la non-violence prônée par Gandhi ou Martin Luther King. À toutes ces questions, les trois articles ont pu apporter des réponses. Ainsi, M. Gardenier et A. Monnier montrent comment deux collectifs vigilantistes anti-migrants cherchent à adoucir leur image afin d'éviter des sanctions juridiques et la fermeture de leurs outils numériques de communication. Pour ce faire, ils insistent sur le recours au logos et modifient les dimensions pathémiques utilisées afin de préserver leur ethos. M. G. Dascalakis-Labreze explore, quant à elle, les stratégies de retournement de stigmate des candidats à la présidentielle argentine, enclins à reprendre à leur compte les catégories visant initialement à les disqualifier. Enfin, È. Gianoncelli explique comment les pensées d'un socialiste (Jean-Claude Michéa) et d'un conservateur proche de l'extrême droite (Gaultier Bès) peuvent converger dans une aspiration à un «nouvel enracinement», jugé essentiel à la société.

\section{Altérité}

À partir de ce premier point, nous avons défini la radicalité par rapport à l'altérité, au sens où elle se nourrit de dissensus, voire de conflit(s). On est en effet toujours politiquement radical par rapport à quelqu'un (altérité), quelque chose d'autre (objet), à quoi s'articule un discours certes argumenté, mais qui peut aussi receler un puissant appel aux émotions, aux affects (Kelsey, 2016; Deleplace éd., 2009). La caractérisation de ces types de discours se pose en termes de typologie et de catégorisation, en les organisant les uns par rapport aux autres. Se pose également, de manière plus ambitieuse, la question d'une structuration type commune, d'un patron qui serait propre, spécifique à ce que 
l'on nomme ici les radicalités politiques. Les trois articles proposés permettent de répondre à certains de ces points. Ainsi, le texte de M. Gardenier et A. Monnier montre que les collectifs vigilantistes anti-migrants « semblent mettre en œuvre une stratégie visant à rendre leurs croyances intersubjectives, c'est-àdire à exercer une influence sur l'opinion publique concernant la gestion des questions migratoires» (p. 65). Le travail d'È. Gianoncelli montre au contraire (p. 33) que les propos du philosophe Jean-Claude Michéa

procède[nt] ainsi à une double déradicalisation de la gauche, à la fois en lui ôtant toute légitimité à incarner la radicalité et en la présentant comme accompagnant l'expérience du déracinement, jugé caractéristique des sociétés contemporaines.

Enfin, le travail de M. G. Dascalakis-Labreze suggère que la force disqualifiante de l'animalisation de l'adversaire prend sens, en Argentine, dans un contexte politique particulier. Celui-ci est en effet caractérisé par une ancienne, mais toujours forte, polarisation du champ politique entre les héritiers revendiqués du péronisme et ses adversaires viscéraux.

Nous avons pu vérifier qu'un certain type de radicalité politique, défini comme tel, à travers un discours donné, émerge toujours en se définissant en opposition à un autre discours. La dimension de l'altérité - ce par rapport à quoi se définit toute radicalité - se révèle ainsi comme la lecture en creux, à construire, de la radicalité : les discours ressentis comme "pro» par certains sont en effet toujours appréhendés comme des discours «anti » par d'autres (par exemple, les discours "pour l'indépendance des traités» sont perçus comme des discours "contre le centralisme »). C'est de cette manière que la dynamique de la vie politique argentine paraît structurée par le virulent antagonisme entre, pour le dire vite, d'une part les néolibéraux pro-Macri et d'autre part les post-péronistes - dont Cristina Kirchner apparaît comme la figure de proue. Cette situation exclut tout rapprochement entre les deux, tout comme elle exclut l'émergence d'une tierce position politique. À l'inverse, les collectifs calaisiens étudiés par M. Gardenier et A. Monnier considèrent les ONG et autres associations d'aide aux migrants comme des associations « pro » migration. Autrement dit, là où se trouvent des défenseurs, se rencontrent également des attaquants, chacun se renvoyant régulièrement la balle d'un discours qui tend toujours, pour «les autres», vers une certaine forme de radicalité, plus ou moins déclarée ou extrême.

Nous avons ainsi également pu vérifier la manière dont la mise en place d'une radicalité politique advient dans un système de confrontation, d'opposition suffisamment forte pour se réclamer d'une forme d'extrémisme ou d'intégrité non négociable, qui peut passer, ou non, par l'action. C'est dans cette perspective que l'article de M. Gardenier et A. Monnier montre que la négociation de la radicalité des groupes étudiés ne se départit pas d'une inconditionnalité du rejet des migrants. C'est également au nom de cette intégrité non 
négociable que les nouveaux conservateurs, héritiers de la Nouvelle droite et d'Alain de Benoist, se réapproprient des références de gauche, ainsi que le constate È. Gianoncelli.

De ce fait, la place de l'autre, le discours sur, autour de l'autre, et le type de communication choisie pour se faire entendre des autres, mais aussi pour parler des autres (à l'exemple des insultes pour parler des femmes politiques - telles celles assimilant, dans le contexte argentin, C. Kirchner à une jument), demeurent extérieurs à ce système défini et, pour ainsi dire, intracommunautaire de radicalité politique. Ainsi, bien qu'ils reposent tous deux sur l'action, voire sur la performance, la différence, par exemple, entre un système de communication comme la non-violence et celui des Femen est visible. L'expression de toute radicalité politique va de pair avec des choix : l'utilisation de certains types de supports (écrire sur des corps, sur des banderoles, sur des murs, sur des affiches...), de certains médiums de communication (en fonction, aussi, des destinataires visés). En d'autres termes, les méthodes communicationnelles participent à la fois d'une recherche d'efficacité comme d'une identité à travers un style communicationnel plus ou moins marqué (régimes illocutoires, esthétiques).

\section{Temporalités}

Être radical, disait Marx, c'est «saisir les choses à la racine» (1982, p. 390). Si la concentration sur la seule étymologie tend à éclipser la sémantique des usages actuels de l'expression, c'est bien à un rapport particulier à la temporalité que nous invite le fait d'aborder le terme de radicalité; celui d'une nostalgie des principes originels que le passage du temps aurait détournés, affaiblis, voire pervertis, et qu'il s'agirait de restaurer dans leur authenticité.

On constate par ailleurs une normalisation d'anciens discours de radicalité politique - que l'on pense simplement au destin du radicalisme politique, de l'intransigeance républicaine qu'il incarne au XIX ${ }^{e}$ siècle jusqu'à la figure du conformisme institutionnel qu'il deviendra sous les IIIe et IVe Républiques, avant de sombrer sous la Ve. La question se posait aussi de savoir s'il existe des types d'argumentation correspondant à une normalisation progressive du discours, ou bien si le sort de tout discours politique radical est nécessairement, à un moment donné, de s’intégrer diachroniquement à un discours normatif. Autrement dit, il s'agit de déterminer si les radicalités politiques sont toujours l'annonce de normes futures, à venir, en construction et, si oui, de voir sous quelles formes cela se manifeste.

Si donc le discours de radicalité semble par définition peu nuancé, vient un moment où la radicalité cesse de l'être, un moment où s'ébauchent un passage, une transition vers la norme. Si l'on considère par exemple le terme nigger, on 
constate qu'il est devenu une expression polémique marquant une radicalité, que celle-ci soit en faveur ou à l'encontre du combat des Africains-Américains (Bonnet, 2011). La question se pose alors de la réappropriation et de la circulation des discours, voire des détournements discursifs opérés d'une radicalité à l'autre (Fracchiolla, 2015), ainsi que conclut È. Gianoncelli en soulignant les limites au bricolage idéologique que constituent les références à Simone Weil ou Jean-Claude Michéa dans la pensée de la Nouvelle droite. Interviennent ici le rapport à l'histoire, le rapport du «moment historique » au «moment discursif» (Moirand, 2007), mais aussi la mémoire collective à travers l'interdiscours.

\section{Finalités}

Il apparaît clairement que la radicalité touche tous les types de causes (animale, environnementale, sociale, ethnique) et fait rarement l'objet d'un projet politique global ; bien plus, les logiques d'intersectionnalité semblent balkaniser davantage les luttes.

Dès lors, quel est l'objectif du discours radical ? Vise-t-il à susciter une adhésion grâce à la persuasion ou est-il seulement un discours qui cherche à attirer, déplacer l'attention sur un objet non commun, à modifier le point de vue? Quel est, en d'autres termes, son rapport au perlocutoire en termes d'actes de langage (Austin, 1962) ? On a pu remarquer également que les radicalités politiques réutilisent d'autres discours, ceux d'autres types de radicalités, en les transformant. Par exemple, en les remettant en circulation, sous d'autres formes, comme la circulation de la rediscursivisation (Mills, 2008) ou encore la forme d'une remise en discours, d'une resignification (si l'on songe au panafricanisme). La dimension sectorielle et la logique d'interpellation supposent une fois de plus la prise en compte de la matérialité langagière, les signifiés et les supports de signification générant des logiques d'identification (les tenues des Black Panthers) ou de reconnaissance (les logiques de cryptisme, notamment par les jeux de mots entre initiés).

L'article de M. G. Dascalakis-Labreze met ainsi en évidence la manière dont les discours radicaux utilisent parfois les cadres de la montée en tension pour provoquer, susciter l'attention - dans une dimension interpellative, donc, et non nécessairement de réelle violence verbale adressée (Moïse, 2012 ; Auger et al., 2008; Fracchiolla etal., 2013). Notamment, la majorité y est en effet interpellée, par l'utilisation d'insultes pour parler de C. Kirchner, à travers des mises en scène discursives et certains procédés rhétoriques spécifiques. Les différentes approches géographiques et temporelles proposées par les articles du dossier ont également permis de voir, dans une certaine mesure, la manière dont les perceptions des radicalités pouvaient varier en fonction des cultures (Barbut, 2012). 
Cette livraison montre ainsi, à travers trois études de cas d’horizons géographiques divers, mais toutes d'une actualité proche, les relations ambivalentes à la radicalité que peuvent avoir des acteurs politiques différents (partis politiques, groupes de citoyens ou intellectuels). Elle s’intéresse à des stratégies de communication ou de positionnement travaillant l'affichage des radicalités : la polémicité, la réappropriation du stigmate induit par celle-ci, ou au contraire l'atténuation du discours; ou encore une référence aux origines permettant de se réapproprier une pensée censément antagoniste. Ces trois articles montrent in fine que la radicalité, qu'elle soit revendiquée ou stigmatisée, qu'elle exige un retour aux origines ou annonce des temps nouveaux, qu'elle mobilise des émotions ou en appelle à la responsabilité, n'est qu'une des manières de dire la conflictualité au cœur du politique.

\section{Références}

Amossy Ruth, 2014, Apologie de la polémique, Paris, PUF.

Austin John Langshaw, 1962, How to Do Things with Words, Oxford, Clarendon Press.

BARBUT Michael, 2012, " “Qui sont les terroristes?” Lutte de classement autour de la radicalité mapuche », Lien social et Politiques, n68, p. 79-100.

BONNET Valérie, 2017, "Malcolm X, the awaken negro», intervention lors du colloque "L'ethos de rupture, de l'Antiquité à nos jours» (Paris, 12-13 octobre 2017).

- 2011, «"Don't call me Nigger, Whitey” : l'autodésignation de la communauté afroaméricaine et la construction identitaire», Communication, vol. XXVIII, n² 2, http:// communication.revues.org/1803 (consulté le 7 avril 2020).

Bonnet Valérie, Geslin Albane éd., 2019, Les mots de l'écologie [numéro thématique], Mots. Les langages du politique, n 119.

Collovald Annie, GAïTı Brigitte éd., 2006, La démocratie aux extrêmes : sur la radicalisation politique, Paris, La Dispute.

DELEPLACE Marc éd., 2009, Les discours de la haine : récits et figures de la passion dans la Cité, Villeneuve d'Ascq, Presses universitaires du Septentrion.

FRACCHIOLLA Béatrice, 2015, "Violence verbale dans le discours des mouvements antagonistes : le cas de "Mariage pour tous" et "Manif pour tous" ", Argumentation et analyse du discours, $\mathrm{n}^{\circ}$ 14, http://aad.revues.org/1940 (consulté le 7 avril 2020).

Fracchiolla Béatrice, Moïse Claudine, Schultz-Romain Christina etal., 2013, Violences verbales: analyses, enjeux et perspectives, Rennes, Presses universitaires de Rennes.

KELSEY Darren, 2016, «Hero mythology and right-wing populism: a discourse-mythological case study of Nigel Farage in the Mail Online», Journalism Studies, vol. XVII, nº 8, p. $971-988$.

MARX Karl, 1982 [1844], Pour une critique de la philosophie du droit de Hegel, dans K. Marx, CEuvres. III, Philosophie, M. Rubel éd., Paris, Gallimard.

MAthieu Lilian, 2003, "L'extrême droite mouvementiste : le cas d'Unité radicale », Contretemps, no8, p. 86-93. 
Milus Sara, 2008, Language and Sexism, Cambridge, Cambridge University Press.

MoIRAND Sophie, 2007, Les discours de la presse quotidienne : observer, analyser, comprendre, Paris, PUF.

MoïsE Claudine, 2012, "Argumentation, confrontation et violence verbale fulgurante», Argumentation et analyse du discours, n 8, https://journals.openedition.org/aad /1260?lang=en (consulté le 7 avril 2020).

Moïse Claudine, AUgER Nathalie, FrAcch Iolla Béatrice et al. éd., 2008, La violence verbale, Paris, L'Harmattan.

PLANTIN Christian, 2016, Dictionnaire de l'argumentation : une introduction aux études d'argumentation, Lyon, ENS Éditions.

QUÉRÉ Louis, 2017, «Les passions tristes du populisme», Occasional Papers, n ${ }^{43}$, Paris, CEMS, http://cems.ehess.fr/docannexe/file/4018/op43.pdf (consulté le 7 avril 2020). 